\title{
MULTI-DIMENSIONAL HADAMARD'S INEQUALITIES
}

\author{
YIN CHEN
}

\begin{abstract}
In this paper, Hadamard's inequalities are extended to a convex function on a convex set in $\mathbf{R}^{2}$ or $\mathbf{R}^{3}$. In particular, it is proved that the average of convex function on a disc of radius $r$ is between the average of the function on the circle of radius $r$ and that on the circle of $\frac{2 r}{3}$.
\end{abstract}

\section{Introduction}

Let $f$ be a convex function defined on $[a, b]$. Then we have the following inequalities, which are called Hermite-Hadamard's inequalities, or simply Hadamard's inequalities,

$$
f\left(\frac{a+b}{2}\right) \leq \frac{1}{b-a} \int_{a}^{b} f(x) d x \leq \frac{f(a)+f(b)}{2} .
$$

We want to extend Hadamard's inequalities to a convex function of several variables. Recall that a function defined on a convex domain $D$ of a vector space is convex if for any nonnegative constant $\alpha \in[0,1]$ and any two points $x_{1}, x_{2} \in D$, the following inequality holds

$$
f\left(\alpha x_{1}+(1-\alpha) x_{2}\right) \leq \alpha f\left(x_{1}\right)+(1-\alpha) f\left(x_{2}\right) .
$$

Let us consider a function $f(x, y)$ on a convex subset $D$ of $\mathbf{R}^{2}$. Note that if $f(x, y)$ is a convex function on $D$, then it is convex on any line segment in $D$ and in particular, it is convex of both $x$ and $y$.

Hadamard's inequalities deal with a convex function on $[a, b]$. It states that the average of a convex function $f$ on $[a, b]$ is between the values of $f$ at the midpoint $x=\frac{a+b}{2}$ and the average of the values of $f$ at the endpoints $a$ and $b$. Let us consider a convex function $f$ on a disk. A nature question is to ask if the similar inequalities hold for the function $f$ on an annulus (contained in the disk): $a \leq r \leq b$ where $r=\sqrt{x^{2}+y^{2}}$. We show that we do have such similar inequalities. A particular case of these inequalities improves an inequality of Hadamard's type on a disk obtained by Chen [2] or Dragomir [3]. We also get some similar inequalities of Hadamard's type for a convex function on a regular polygon. We extend our 
results then to a convex function on a ball of $\mathbf{R}^{3}$. The reader is referred to [5] and [6] for the original papers on Hadamard's inequality, and [1], [4], [7], [8], [9], [10] or [13] for some new developments on this topic.

\section{Hadamard's inequality on an annulus}

In this section, we will fix the following notations

$$
D(r)=\left\{(x, y): x^{2}+y^{2} \leq r^{2}\right\}
$$

and

$$
L(r)=\left\{(x, y): x^{2}+y^{2}=r^{2}\right\}
$$

We will always consider a convex function on a disk centred at $(0,0)$. It is easy to see that our inequalities will be true for a convex function on a disk centred at any point in $\mathbf{R}^{2}$. Let $f$ be a convex function on $D(R)$. For $0 \leq r \leq R$, we define

$$
\begin{aligned}
& F(r, \theta)=f(r \cos \theta, r \sin \theta), \\
& M_{f}(r)=\sup \left\{f(x, y): x^{2}+y^{2}=r^{2}\right\}, \\
& C_{f}(r)=\frac{1}{2 \pi r} \int_{L(r)} f(x, y) d s,
\end{aligned}
$$

and

$$
B_{f}(r)=\frac{1}{\pi r^{2}} \iint_{D(r)} f(x, y) d x d y .
$$

Clearly

$$
C_{f}(r)=\frac{1}{2 \pi} \int_{0}^{2 \pi} F(r, \theta) d \theta
$$

and

$$
\begin{aligned}
B_{f}(r) & =\frac{1}{\pi} \int_{0}^{1} \int_{0}^{2 \pi} F(r t, \theta) t d \theta d t \\
& =2 \int_{0}^{1} C_{f}(r t) t d t .
\end{aligned}
$$

The following theorem is an analogue of Theorem 2.6.8 of [11] for the convex functions.

Theorem 2.1. Let $f$ be a convex function on $D(R)$. Then the functions $M_{f}(r), C_{f}(r)$ and $B_{f}(r)$ are all increasing convex functions of $r$ on $[0, R]$.

Proof. We first observe that $F(r, \theta)$ is a convex function of $r$ on $[0, R]$ for any $\theta \in[0,2 \pi]$. Thus for any nonnegative constants $\alpha_{1}$ and $\alpha_{2}$ with $\alpha_{1}+\alpha_{2}=1$ and for any $r_{1}, r_{2} \in[0, R]$, we have

$$
F\left(\alpha_{1} r_{1}+\alpha_{2} r_{2}, \theta\right) \leq \alpha_{1} F\left(r_{1}, \theta\right)+\alpha_{2} F\left(r_{2}, \theta\right)
$$


Taking the supremum for $\theta$ over $[0,2 \pi]$ on both sides of the above inequality gives the convexity of $M_{f}(r)$. If we integrate both sides of (9) for $\theta$ over [0,2 $\pi$, and then divide both sides by $2 \pi$, we know that $C_{f}(r)$ is convex. If we replace $r_{1}$ and $r_{2}$ by $r_{1} t$ and $r_{2} t$ in (9), multiply $\frac{t}{\pi}$ on both sides, and then integrate both sides for $t$ over $[0,1]$ and for $\theta$ over $[0,2 \pi]$, we will have that $B_{f}(r)$ is also convex.

Let $r_{1}, r_{2}$ be two constants such that $0 \leq r_{1} \leq r_{2} \leq R$. Note that

$$
r_{1}=\left(\frac{1}{2}+\frac{r_{1}}{2 r_{2}}\right) r_{2}+\left(\frac{1}{2}-\frac{r_{1}}{2 r_{2}}\right)\left(-r_{2}\right) .
$$

Since the three points $\left(r_{2}, \theta\right),\left(r_{1}, \theta\right)$ and $\left(r_{2}, \theta+\pi\right)$ are on the same line and $f$ is convex on the line, by (10), we have

$$
F\left(r_{1}, \theta\right) \leq\left(\frac{1}{2}+\frac{r_{1}}{2 r_{2}}\right) F\left(r_{2}, \theta\right)+\left(\frac{1}{2}-\frac{r_{1}}{2 r_{2}}\right) F\left(r_{2}, \theta+\pi\right) .
$$

Taking supremum on both sides of (11) for $\theta \in[0,2 \pi]$ gives that

$$
M_{f}\left(r_{1}\right) \leq\left(\frac{1}{2}+\frac{r_{1}}{2 r_{2}}\right) M_{f}\left(r_{2}\right)+\left(\frac{1}{2}-\frac{r_{1}}{2 r_{2}}\right) M_{f}\left(r_{2}\right)=M_{f}\left(r_{2}\right) \text {. }
$$

So $M_{f}(r)$ is increasing.

If we take integral on both sides of (11) for $\theta \in[0,2 \pi]$ and multiply both sides by $\frac{1}{2 \pi}$, we have

$$
C_{f}\left(r_{1}\right) \leq\left(\frac{1}{2}+\frac{r_{1}}{2 r_{2}}\right) C_{f}\left(r_{2}\right)+\left(\frac{1}{2}-\frac{r_{1}}{2 r_{2}}\right) C_{f}\left(r_{2}\right)=C_{f}\left(r_{2}\right) .
$$

This shows that $C_{f}(r)$ are increasing on $[0, R]$.

If we replace $r_{1}$ and $r_{2}$ by $t r_{1}$ and $t r_{2}$ in (11), multiply both sides by $\frac{t}{\pi}$, and then do the integrals for $t$ over $[0,1]$ and for $\theta$ over $[0,2 \pi]$, we have

$$
B_{f}\left(r_{1}\right) \leq\left(\frac{1}{2}+\frac{r_{1}}{2 r_{2}}\right) B_{f}\left(r_{2}\right)+\left(\frac{1}{2}-\frac{r_{1}}{2 r_{2}}\right) B_{f}\left(r_{2}\right)=B_{f}\left(r_{2}\right) .
$$

This shows that $B_{f}(r)$ is also increasing.

Now let us consider Hadamard's inequalities over $a \leq r \leq b$.

Theorem 2.2. Let $f(x, y)$ be a convex function on the disk $D(R)$. If $0 \leq a<b \leq R$, then

$$
\begin{aligned}
& \frac{1}{\pi\left(b^{2}-a^{2}\right)} \iint_{a \leq r \leq b} f(x, y) d x d y \geq C_{f}\left(\frac{2\left(a^{2}+a b+b^{2}\right)}{3(a+b)}\right) \\
& \frac{1}{\pi\left(b^{2}-a^{2}\right)} \iint_{a \leq r \leq b} f(x, y) d x d y \leq \frac{2 a+b}{3(a+b)} C_{f}(a)+\frac{a+2 b}{3(a+b)} C_{f}(b) .
\end{aligned}
$$


Proof. For any $\theta \in[0,2 \pi], F(r, \theta)$ is a convex function of $r$ on $[0, R]$. So there is a constant $A(\theta)$ not depending on $r$ such that

$$
F(r, \theta) \geq F\left(r_{0}, \theta\right)+A(\theta)\left(r-r_{0}\right)
$$

where $r_{0}=\frac{2\left(a^{2}+a b+b^{2}\right)}{3(a+b)}$. Therefore

$$
\begin{aligned}
\iint_{a \leq r \leq b} f(x, y) d x d y & =\int_{0}^{2 \pi} \int_{a}^{b} F(r, \theta) r d r d \theta \\
& \geq \int_{0}^{2 \pi} \int_{a}^{b} F\left(r_{0}, \theta\right) r d r d \theta+\int_{0}^{2 \pi} \int_{a}^{b} A(\theta)\left(r^{2}-r_{0} r\right) d r d \theta \\
& =\frac{b^{2}-a^{2}}{2} \int_{0}^{2 \pi} F\left(r_{0}, \theta\right) d \theta+\int_{0}^{2 \pi} 0 d \theta \\
& =\pi\left(b^{2}-a^{2}\right) C_{f}\left(r_{0}\right) .
\end{aligned}
$$

This proves the first inequality.

To show the second inequality, we first note that

$$
F(r, \theta) \leq \frac{b-r}{b-a} F(a, \theta)+\frac{r-a}{b-a} F(b, \theta),
$$

for any $r: a \leq r \leq b$ and any $\theta \in[0,2 \pi]$, since $F(r, \theta)$ is convex function of $r$. It follows that

$$
\begin{aligned}
& \frac{1}{\pi\left(b^{2}-a^{2}\right)} \iint_{a \leq r \leq b} f(x, y) d x d y \\
& \quad \leq \frac{1}{\pi\left(b^{2}-a^{2}\right)} \int_{0}^{2 \pi} \int_{a}^{b}\left[\frac{b-r}{b-a} r F(a, \theta)+\frac{r-a}{b-a} r F(b, \theta)\right] d r d \theta \\
& \quad \leq \frac{2 a+b}{3(a+b)} C_{f}(a)+\frac{a+2 b}{3(a+b)} C_{f}(b) .
\end{aligned}
$$

This completes the proof.

Letting $a \rightarrow 0$, we have Hadamard's inequalities on a disk.

Corollary 2.3. Let $f$ be a convex function on $D(R)$ and $0 \leq b<R$. Then

$$
C_{f}\left(\frac{2}{3} b\right) \leq B_{f}(b) \leq \frac{1}{3} f(0,0)+\frac{2}{3} C_{f}(b) .
$$

By Theorem (2.1), we know that $f(0,0) \leq C_{f}(b)$, thus Corollary (2.3) implies and improves the following Hadamard's inequality on a disk, obtained by Dragomir [3] and also by Chen [2].

Corollary 2.4. Let $f$ be a convex function on $[0, R]$. Then

$$
f(0,0) \leq C_{f}\left(\frac{2}{3} b\right) \leq B_{f}(b) \leq C_{f}(b) \leq M_{f}(b) .
$$


Let $f(x, y)=\sqrt{x^{2}+y^{2}}$. Then $f$ is a convex function on $\mathbf{R}^{2}$. It is easy to find $M_{f}(r)=r$, $C_{f}(r)=r$ and $B_{f}(r)=\frac{2}{3} r$. Therefore the constant $\frac{2}{3}$ on both sides of (12) is the best possible.

\section{Hadamrad's inequalities and centroid}

Let $(\bar{x}, \bar{y})$ be the centroid of a convex set $D$ in $\mathbf{R}^{2}$. Let us compare the average value of $f$ on $D$ with the value of $f$ at the centriod.

Theorem 3.1. Let $f$ be a convex function on a convex region $D \subset \mathbf{R}^{2}$. Then

$$
f(\bar{x}, \bar{y}) \leq \frac{1}{A(D)} \iint_{D} f(x, y) d x d y
$$

where $(\bar{x}, \bar{y})$ is the centroid of $D$ and $A(D)$ is the area of $D$.

Proof. Since $(\bar{x}, \bar{y})$ is the centroid of $D$, we have

$$
\bar{x}=\frac{\iint_{D} x d x d y}{\iint_{D} d x d y}, \quad \bar{y}=\frac{\iint_{D} y d x d y}{\iint_{D} d x d y} .
$$

It follows that

$$
\iint_{D}(x-\bar{x}) d x d y=0, \quad \iint_{D}(y-\bar{y}) d x d y=0 .
$$

$f$ being a convex function on $D$ implies that $f$ has support at $(\bar{x}, \bar{y})$ ([12], page 108), that is

$$
f(x, y) \geq f(\bar{x}, \bar{y})+A(x-\bar{x})+B(y-\bar{y})
$$

for some constants $A$ and $B$ and for any $(x, y) \in D$. Therefore

$$
\begin{aligned}
\iint_{D} f(x, y) d x d y & \geq \iint_{D}(f(\bar{x}, \bar{y})+A(x-\bar{x})+B(y-\bar{y})) d x d y \\
& =\iint_{D} f(\bar{x}, \bar{y}) d x d y \quad(\text { by (15)) } \\
& =f(\bar{x}, \bar{y}) A(D),
\end{aligned}
$$

which completes the proof.

\section{Hadamrad's inequality on a regular polygon}

We now give an application to Theorem (3.1).

We see in section 2 that the average of a convex function on a disk is between the average of the function on the boundary and the average of the function on a shrunk curve to $\frac{2}{3}$ size of the boundary. We will show that this is true too for a convex function on a regular polygon.

First we need a lemma for the convex function on a triangle. Let $\Delta$ be a triangle and $A, B, C$ be three vertices. Take points $E$ and $F$ on line segments $A B$ and $A C$ respectively such that $|A E|=\frac{2}{3}|A B|$ and $|A F|=\frac{2}{3}|A C|$. Then 


\section{Lemma 4.1.}

$$
\begin{aligned}
& \frac{1}{A(\Delta)} \iint_{\Delta} f(x, y) d x d y \geq \frac{1}{|E F|} \int_{E F} f(x, y) d s \\
& \frac{1}{A(\Delta)} \iint_{\Delta} f(x, y) d x d y \leq \frac{1}{3} f\left(x_{A}, y_{A}\right)+\frac{2}{3|B C|} \int_{B C} f(x, y) d s
\end{aligned}
$$

where $\left(x_{A}, y_{A}\right)$ is the coordinates of point $A$, and $A(\Delta)$ is the area of $\Delta$.

Proof. Without loss of generality, let us consider a triangle $A B C$ such that $B$ is at $(0,0), C$ is at $(p, 0)$ with $p>0$ and $A$ is at $\left(x_{A}, y_{A}\right)$. Let $n$ be a positive integer larger than 2 . Let $D_{0}$ be the point $B, D_{n}$ be the point $C$ and $D_{i}$ be the points $\left(\frac{i}{n} p, 0\right)$ for $i=1,2, \ldots, n-1$. Clearly for $i=$ $1,2, \ldots, n$, the small triangle $A D_{i-1} D_{i}$ has the area $\frac{A(\Delta)}{n}$ and has the centroid $\left(\frac{2 i-1}{3 n} p+\frac{x_{A}}{3}, \frac{y_{A}}{3}\right)$. Applying Theorem (3.1) on each triangle $A D_{i-1} D_{i}$, we have

$$
\frac{n}{A(\Delta)} \iint_{\Delta\left(A D_{i-1} D_{i}\right)} f(x, y) d x d y \geq f\left(\frac{2 i-1}{3 n} p+\frac{x_{A}}{3}, \frac{y_{A}}{3}\right) .
$$

Adding both sides for $i=1,2, \ldots, n$, we have

$$
\frac{n}{A(\Delta)} \iint_{\Delta} f(x, y) d x d y \geq \sum_{i=1}^{n} f\left(\frac{2 i-1}{3 n} p+\frac{x_{A}}{3}, \frac{y_{A}}{3}\right),
$$

or

$$
\begin{aligned}
\frac{1}{A(\Delta)} \iint_{\Delta} f(x, y) d x d y & \geq \sum_{i=1}^{n} \frac{1}{n} f\left(\frac{2 i-1}{3 n} p+\frac{x_{A}}{3}, \frac{y_{A}}{3}\right) \\
& =\frac{3}{2 p} \sum_{i=1}^{n} \frac{2 p}{3 n} f\left(\frac{2 i-1}{3 n} p+\frac{x_{A}}{3}, \frac{y_{A}}{3}\right) \\
& =\frac{1}{|E F|} \sum_{i=1}^{n} \frac{2 p}{3 n} f\left(\frac{2 i-1}{3 n} p+\frac{x_{A}}{3}, \frac{y_{A}}{3}\right) .
\end{aligned}
$$

As $n \rightarrow \infty$, clearly last sum goes to the integral of $f$ on $E F$

$$
\begin{aligned}
\lim _{n \rightarrow \infty} \frac{1}{|E F|} \sum_{i=1}^{n} \frac{2 p}{3 n} f\left(\frac{2 i-1}{3 n} p+\frac{x_{A}}{3}, \frac{y_{A}}{3}\right) & \left.=\frac{1}{|E F|} \int_{\frac{x_{A}}{3}}^{\frac{x_{A}+2 p}{3}} f\left(x, \frac{y_{A}}{3}\right)\right) d x \\
& =\frac{1}{|E F|} \int_{E F} f(x, y) d s .
\end{aligned}
$$

This proves (16).

To prove (17), we will use a theorem in [1] that says the average of a convex function on a triangle is less than or equal to the average of the values of the function at the three vertices. Applying this theorem on each small triangle $A D_{i-1} D_{i}$ for $i=1,2, \ldots, n$, we have

$$
\frac{n}{A(\Delta)} \iint_{\Delta\left(A D_{i-1} D_{i}\right)} f(x, y) d x d y \leq \frac{f\left(x_{A}, y_{A}\right)+f\left(\frac{i-1}{n} p, 0\right)+f\left(\frac{i}{n} p, 0\right)}{3} .
$$


Adding all these inequalities yields

$$
\frac{n}{A(\Delta)} \iint_{\Delta} f(x, y) d x d y \leq \sum_{i=1}^{n} \frac{f\left(x_{A}, y_{A}\right)+f\left(\frac{i-1}{n} p, 0\right)+f\left(\frac{i}{n} p, 0\right)}{3}
$$

or

$$
\begin{aligned}
\frac{1}{A(\Delta)} \iint_{\Delta} f(x, y) d x d y & \leq \sum_{i=1}^{n} \frac{f\left(x_{A}, y_{A}\right)+f\left(\frac{i-1}{n} p, 0\right)+f\left(\frac{i}{n} p, 0\right)}{3 n} \\
& =\frac{f\left(x_{A}, y_{A}\right)}{3}+\sum_{i=1}^{n} \frac{f\left(\frac{i-1}{n} p, 0\right)+f\left(\frac{i}{n} p, 0\right)}{3 n} \\
& =\frac{f\left(x_{A}, y_{A}\right)}{3}+\frac{f(0,0)-f(p, 0)}{3 n}+\frac{2}{3 p} \sum_{i=1}^{n} \frac{p}{n} f\left(\frac{i}{n} p, 0\right) .
\end{aligned}
$$

Clearly, as $n \rightarrow \infty$, the above sum has a limit

$$
\lim _{n \rightarrow \infty} \sum_{i=1}^{n} \frac{1}{n} f\left(\frac{i}{n} p, 0\right)=\int_{0}^{p} f(x, 0) d x=\int_{B C} f(x, y) d s .
$$

This proves (17).

Now we will extend these inequalities to a convex function on a regular polygon.

Theorem 4.2. Let $f$ be convex on a regular $n$-gon $P_{n}, C(a, b)$ be the center of $P_{n}, D_{n}$ be the boundary of $P_{n}$ and $E_{n}$ be the boundary of the $n$-gon whose vertices are the points on the line segments connecting $C$ and the vertices of $P_{n}$ and $\frac{2}{3}$ of the length of the segments from $C$. Then

$$
\begin{aligned}
& \frac{1}{A\left(P_{n}\right)} \iint_{P_{n}} f(x, y) d x d y \geq \frac{1}{\left|E_{n}\right|} \int_{E_{n}} f(x, y) d s \\
& \frac{1}{A\left(P_{n}\right)} \iint_{P_{n}} f(x, y) d x d y \leq \frac{1}{3} f(a, b)+\frac{2}{3\left|D_{n}\right|} \int_{D_{n}} f(x, y) d s
\end{aligned}
$$

Proof. Divide the $n$-gon to $n$ identical triangles and apply Lemma (4.1) to each triangle.

If $n \rightarrow \infty$ in (18) and (19), then we can get another proof for Corollary (2.3).

\section{Three dimensional Hadamard's inequalities}

Let $B(r)$ and $S(r)$ be the ball and the surface of the ball of radius $r$ in the space, that is,

$$
B(r)=\left\{(x, y, z): x^{2}+y^{2}+z^{2} \leq r^{2}\right\}
$$

and

$$
S(r)=\left\{(x, y, z): x^{2}+y^{2}+z^{2}=r^{2}\right\} .
$$

Let $f$ be a convex function on $D(R)$. For $0 \leq r \leq R$, we define

$$
F(\rho, \phi, \theta)=f(\rho \sin \phi \cos \theta, \rho \sin \phi \sin \theta, \rho \cos \phi),
$$




$$
\begin{aligned}
& M_{f}(r)=\sup \left\{f(x, y, z): x^{2}+y^{2}+z^{2}=r^{2}\right\}, \\
& Q_{f}(r)=\frac{1}{4 \pi r^{2}} \iint_{S(r)} f(x, y, z) d S,
\end{aligned}
$$

and

$$
P_{f}(r)=\frac{3}{4 \pi r^{3}} \iiint_{B(r)} f(x, y, z) d x d y d z
$$

Clearly

$$
Q_{f}(r)=\frac{1}{4 \pi} \int_{0}^{\pi} \int_{0}^{2 \pi} F(r, \phi, \theta) \sin \phi d \theta d \phi,
$$

and

$$
\begin{aligned}
P_{f}(r) & =\frac{3}{4 \pi} \int_{0}^{1} \int_{0}^{\pi} \int_{0}^{2 \pi} F(r t, \theta) t \sin \phi d t d \theta d \phi d t \\
& =3 \int_{0}^{1} Q_{f}(r t) t^{2} d t .
\end{aligned}
$$

By the similar way as in the proof of Theorem (2.1), we can easily prove the following theorem.

Theorem 5.1. Let $f$ be a convex function on $B(R)$. Then the functions $M_{f}(r), P_{f}(r)$ and $Q_{f}(r)$ are all increasing convex functions of $r$ on $[0, R]$.

Theorem 5.2. Let $f$ be a convex function on $B(R)$ and $0 \leq b<R$. Then

$$
Q_{f}\left(\frac{3}{4} b\right) \leq P_{f}(b) \leq \frac{1}{4} f(0,0,0)+\frac{3}{4} Q_{f}(b) .
$$

Proof. Let $\rho_{0}=\frac{3}{4} b$. Since $F(\rho, \phi, \theta)$ is convex of $\rho$ on $[0, R]$, it follows that there is $A(\phi, \theta)$ such that

$$
F(\rho, \phi, \theta) \geq F\left(\rho_{0}, \phi, \theta\right)+A(\phi, \theta)\left(\rho-\rho_{0}\right) .
$$

Clearly

$$
\int_{0}^{b} \int_{0}^{\pi} \int_{0}^{2 \pi} A(\phi, \theta)\left(\rho-\rho_{0}\right) \rho^{2} \sin \phi d \theta d \phi d \rho=0 .
$$

By (20) and (21), we will have

$$
\begin{aligned}
P_{f}(b) & =\frac{3}{4 \pi b^{3}} \int_{0}^{\pi} \int_{0}^{2 \pi} \int_{0}^{b} F(\rho, \phi, \theta) \rho^{2} \sin \phi d \rho d \theta d \phi \\
& \geq \frac{3}{4 \pi b^{3}} \int_{0}^{\pi} \int_{0}^{2 \pi} \int_{0}^{b} F\left(\rho_{0}, \phi, \theta\right) \rho^{2} \sin \phi d \rho d \theta d \phi \\
& =\frac{1}{4 \pi} \int_{0}^{\pi} \int_{0}^{2 \pi} F\left(\rho_{0}, \phi, \theta\right) \sin \phi d \theta d \phi \\
& =Q_{f}\left(\rho_{0}\right) .
\end{aligned}
$$


On the other hand, since

$$
\rho \leq\left(1-\frac{\rho}{b}\right) 0+\frac{\rho}{b} b
$$

we have

$$
F(\rho, \phi, \theta) \leq\left(1-\frac{\rho}{b}\right) f(0,0,0)+\frac{\rho}{b} F(b, \phi, \theta)
$$

Thus

$$
\begin{aligned}
P_{f}(b) \leq & \frac{3}{4 \pi b^{3}} \int_{0}^{\pi} \int_{0}^{2 \pi} \int_{0}^{b} F(\rho, \phi, \theta) \rho^{2} \sin \phi d \rho d \theta d \phi \\
\geq & \frac{3}{4 \pi b^{3}} \int_{0}^{\pi} \int_{0}^{2 \pi} \int_{0}^{b}\left(1-\frac{\rho}{b}\right) f(0,0,0) \rho^{2} \sin \phi d \rho d \theta d \phi \\
& +\frac{3}{4 \pi b^{3}} \int_{0}^{\pi} \int_{0}^{2 \pi} \int_{0}^{b} \frac{\rho}{b} F(b, \phi, \theta) \rho^{2} \sin \phi d \rho d \theta d \phi \\
= & \frac{1}{4} f(0,0,0)+\frac{3}{4} Q_{f}(b) .
\end{aligned}
$$

Using the same way in the proof of Theorem (3.1), we can easily see the following theorem.

Theorem 5.3. Let $f$ be a convex function on a convex domain $D \subset \mathbf{R}^{3}$. Then

$$
f(\bar{x}, \bar{y}, \bar{z}) \leq \frac{1}{V(D)} \iiint_{D} f(x, y, z) d x d y d z
$$

where $(\bar{x}, \bar{y}, \bar{z})$ is the centroid of $D$ and $V(D)$ is the volume of $D$.

\section{References}

[1] M. Bessenyei, The Hermite-Hadamard inequality on simplices, Amer. Math. Monthly, 115 (2008), No. 4, 339345 .

[2] Y. Chen, Hadamard's inequality on a polygon, Tamkang J. Math., 35 (2004), No.3, 247-254.

[3] S. S. Dragomir, On Hadamard's inequality on a disk, JIPAM. J. Inequal. Pure Appl. Math., 1(2000), no. 2, Article 2, $11 \mathrm{pp}$.

[4] B. Gavrea, On Hadamard's inequality for the convex mappings defined on a convex domain in the space, $J$. Inequal. Pure Appl. Math., 1 (2000), Article 9.

[5] J. Hadamard, Étude sur les propriétés des fonctions entières et en particulier d'une fonction considérée par Riemann, J. Math. Pures Appl., 58 (1893), 171-215.

[6] Ch. Hermite, Sur deux limites d'une integrale define, Mathesis, 3 (1883), 82.

[7] L. Matejíćka, Elementary proof of the left multidimensional Hermite-Hadamard inequality on certain convex sets, J. Math. Inequal., 4 (2010), no. 2, 259-270.

[8] D. S. Mitrinović and I. B. Lacković, Hermite and convexity, Aequationes Math., 28 (1985), 229-232.

[9] C. P. Niculescu, The Hermite-Hadamard inequality for convex functions of a vector variable, Math. Inequal. Appl., 5 (2002), 619-623.

[10] C. P. Niculescu and L.-E. Persson, Old and new on the Hermite-Hadamard inequality, Real Anal. Exchange, 29 (2003/2004) 619-623.

[11] T. Ransford, Potential Theory In The Complex Plane, Cambridge University Press, 1995. 
[12] A. W. Robert and D. E. Varberg, Convex Functions, Academic Press, New York, 1973.

[13] T. Trif, Characterizations of convex functions of a vector variable via Hermite-Hadamard's inequality, J. Math. Inequal., 1 (2008), No. 2, 37-44.

Department of Mathematical Sciences, Lakehead University, Thunder Bay, Ontario, Canada, P7B 5E1.

E-mail: yin.chen@lakeheadu.ca 